\title{
On Solving Bi-level Multi-Objective Fully Quadratic Fractional Optimization Model with Fuzzy Demands
}

\author{
Namrata Rani \\ Department of Mathematics, \\ Maharishi Markandeshwar (Deemed to be University), Mullana-Ambala, India. \\ E-mail: namratagarg55@gmail.com \\ Vandana Goyal \\ Department of Mathematics, \\ Maharishi Markandeshwar (Deemed to be University), Mullana-Ambala, India. \\ E-mail: vandanagoya104@gmail.com \\ Deepak Gupta \\ Department of Mathematics, \\ Maharishi Markandeshwar (Deemed to be University), Mullana-Ambala, India. \\ Corresponding author: guptadeepak20003@gmail.com
}

(Received on May 28, 2021; Accepted on October 19, 2021)

\begin{abstract}
This paper has been designed to introduce the method for solving the Bi-level Multi-objective (BL-MO) Fully Quadratic Fractional Optimization Model through Fuzzy Goal Programming (FGP) approach by utilising non-linear programming. In Fully Quadratic Fractional Optimization Model, the objective functions are in fractional form, having quadratic functions in both numerator and denominator subject to quadratic constraints set. The motive behind this paper is to provide a solution to solve the BL-MO optimization model in which number of decision-makers (DM) exists at two levels in the hierarchy. First, the fractional functions with fuzzy demand, which are in the form of fuzzy numbers, are converted into crisp models by applying the concept of $\alpha$-cuts. After that, membership functions are developed which are corresponding to each decision-maker's objective and converted into simpler form to avoid complications due to calculations. Finally, the model is simplified by applying FGP approach, and a compromised solution to the initial model is obtained. An algorithm, flowchart and example are also given at the end to explain the study of the proposed model.
\end{abstract}

Keywords- $\alpha$-cut set, BL-MO optimization model, Fuzzy programming, Fully quadratic fractional programming.

\section{Introduction}

Bi-level Multi-objective Fully Quadratic Fractional Optimization Model (BL-MOFQFOM) mostly arises in supply chains, agriculture, traffic control systems, biofuel production, and in many more cases. BL-MOFQFOM is a kind of decision structure in which more than one decision-makers are present at two levels. First level decision-makers are called leaders and followers are the decision-makers on the second level, subject to quadratic constraints. BLMOFQFOM is a demanding tool which has its utilization in the fields of banking, economic systems, healthcare planning, transportation, profit/cost, debt/equity, and so on. A number of objectives emerge in such models, all of which are proposed to be optimized to the higher extent possible. But they agree to compromise up to a certain limit so that every objective function can obtain a satisfactory solution. 
The plan for a two-level programming problem was introduced by Candler and Townsley (1982) in linear objectives. A sequential solution to bi-level linear programming is given by Judice and Faustino (1992). Many methodologies have been developed by many researchers (Shih et al., 1996; Ammar, 2008; Baky, 2009; Baky, 2010; Baky et al., 2014; Osman et al., 2018c; Elsisy and El Sayed, 2019) over the past few decades. Mishra and Ghosh (2006) and Pal and Moitra (2003) have also provided an algorithm to obtain quadratic BL programming problems. In present scenario, the study of bi-level multi-objective is becoming a keen topic among researchers due to its power to solve many practical problems such as in co-operative sectors, closing-out sales, basic cost/target cost, and so on. Abo-Sinna and Baky (2007) has given multiple methods to solve ML programming problems. Osman et al. (2018a) solved various ML models using the FGP approach. Kumar and Rakshit (2017). proposed a methodology for tri-level quadratic fractional programming by using interactive fuzzy programming and solved fractional programming problems with negative semi-definite denominators and introduced two crisp models through upper and lower approximation sets and applied the FGP approach after transformation of variables. Pradhan and Biswal (2019) integrate classical Charnes-Cooper transformation and Lagrange's interpolating polynomial to solve the multi-choice linear fractional programming problem.

In BL-MOFQFOM, the objective functions are quadratic in the numerator and denominator at both levels. In this model, every objective function is interested in self-optimization. Therefore, the focus is to obtain a compromised solution that is admissible to every objective. In this article, the FGP approach is being used to obtain the most effective compromised solution for a number of objective functions present at two levels with coefficients as fuzzy numbers. These fuzzy numbers are intended to be used in the way that the Zadeh (1965) fuzzy set theory specifies. Crisp objective functions with crisp constraints are obtained by using $\alpha$-acts. Membership functions are converted into non-linear form to avoid computational complexity. After that, BL-MOFQFOM is converted into a final model in which under-deviational variables are reduced by extending the work of Osman et al. (2018b).

Reviewing the literature, all the work has been done on linear fractional and linear objective models. But there are situations where we get quadratic fractional objectives. So, the necessity to tackle BL-MOFQFOM with an efficient technique leads us to introduce this paper.

Till now, researchers have been using Taylor's series or parametric approach to get non-fractional models and ignoring round-off and calculation errors that happen due to neglecting small terms. So, a new method has been introduced here to solve BL-MOFQFOM.

The proposed method can be applied to supply chain networks where the first level objectives are to deliver maximum number of products to retailers as well as wholesalers, and the second level objectives are to deliver the order in the shortest possible time.

\section{Preliminaries}

In this paper, the definitions given in Osman et al. (2018b) are used and following theorem is introduced on which our method is based.

Theorem: Let $\tilde{f}(x)=\frac{\tilde{f}_{l}(x)}{\tilde{f}_{2}(x)}$ be a function in Fuzzy environment $\tilde{f}_{1} \in F\left(R_{+}\right)$and $\tilde{f}_{2} \in F\left(R_{++}\right)$ then for maximization of $\tilde{f}, \tilde{f}_{1}$ and $\tilde{f}_{2}$ will be changed by supremum and infimum of $\alpha$-cuts 
respectively. And in minimization of $\tilde{f}, \tilde{f}_{1}$ and $\tilde{f}_{2}$ will be changed by infimum and supremum of $\alpha$-cuts i.e.

$\max \tilde{f}(x)=\max \frac{\tilde{f}_{1}(x)}{\tilde{f}_{2}(x)}$ will become $\frac{U_{\alpha}\left(\tilde{f}_{1}\right)(x)}{L_{\alpha}\left(\tilde{f}_{2}\right)(x)} ; \min \tilde{f}(x)=\min \frac{\tilde{f}_{1}(x)}{\tilde{f}_{2}(x)}$ will become $\frac{L_{\alpha}\left(\tilde{f}_{1}\right)(x)}{U_{\alpha}\left(\tilde{f}_{2}\right)(x)}$, where, $U_{\alpha}$ and $L_{\alpha}$ denotes the sup and inf of $\alpha$-cut of fuzzy parameters.

Proof: Let $\tilde{f}(x)=\frac{\tilde{f}_{1}(x)}{\tilde{f}_{2}(x)}=\frac{x^{T} \tilde{C} x+\tilde{E} x+\tilde{\gamma}}{x^{T} \tilde{D} x+\tilde{F} x+\tilde{\beta}} ; x=\left(x_{1}, x_{2}, \ldots, x_{n}\right)$,

$$
\tilde{C}=\left[\tilde{c}_{i j}\right]_{n \times n}, \tilde{E}=\left[\tilde{e}_{i j}\right]_{1 \times n}, \tilde{D}=\left[\tilde{d}_{i j}\right]_{n \times n}, \tilde{F}=\left[\tilde{f}_{i j}\right]_{1 \times n}
$$

where, the number of the form $\tilde{a}$ is the fuzzy parameter and $\alpha \in[0,1]$.

$\alpha$-cut is defined as:

$(\tilde{b})_{\alpha}=\left\{b \in X \mid \mu_{\tilde{b}}(x) \geq \alpha\right\}=\left[L_{\alpha}(\tilde{b}), U_{\alpha}(\tilde{b})\right]$

then if we want to maximize $\tilde{f}(x)$ will be equivalent to $\max (\tilde{f}(x))_{\alpha}$

$=\max \left(\frac{\tilde{f}_{1}(x)}{\tilde{f}_{2}(x)}\right)_{\alpha}=\max \left(\tilde{f}_{1}(x)\right)_{\alpha} \cdot \max \left(\frac{1}{\tilde{f}_{2}(x)}\right)_{\alpha}=\max \left(\tilde{f}_{1}(x)\right)_{\alpha} \cdot \min \left(\tilde{f}_{2}(x)\right)_{\alpha}$.

So now we are left with two requirements which are $\max \left(\tilde{f}_{1}\right)_{\alpha}$ and $\min \left(\tilde{f}_{2}\right)_{\alpha}$. For this we will prove condition for maximization of $\left(\tilde{f}_{1}(x)\right)_{\alpha}$. Now

$$
\left(\tilde{f}_{1}\right)_{\alpha}=\left(x^{t} \tilde{C} x+\tilde{E} x+\tilde{\gamma}\right)_{\alpha}=\left(x^{T} \tilde{C} x\right)_{\alpha}+(\tilde{E} x)_{\alpha}+(\tilde{\gamma})_{\alpha}=x^{t}(\tilde{C})_{\alpha} x+(\tilde{E})_{\alpha} x+(\tilde{\gamma})_{\alpha}
$$

where,

$$
(\tilde{C})_{\alpha}=\left[L_{\alpha}(\tilde{C}), U_{\alpha}(\tilde{C})\right],(\tilde{E})_{\alpha}=\left[L_{\alpha}(\tilde{E}), U_{\alpha}(\tilde{E})\right],(\tilde{\gamma})_{\alpha}=\left[L_{\alpha}(\tilde{\gamma}), U_{\alpha}(\tilde{\gamma})\right]
$$

From the definition of upper bound, we have $(\tilde{C})_{\alpha} \leq U_{\alpha}(\tilde{C})$

$\Rightarrow x^{t}(\tilde{C})_{\alpha} x \leq x^{t} U_{\alpha}(\tilde{C}) x \quad$ as $\quad x_{1}, x_{2}, \ldots, x_{n} \geq 0 \quad$. Similarly, $(\tilde{E})_{\alpha} \leq U_{\alpha}(\tilde{E}) \Rightarrow(\tilde{E})_{\alpha} x \leq U_{\alpha}(\tilde{E}) x$, $(\tilde{\gamma})_{\alpha} \leq U_{\alpha}(\tilde{\gamma})$.

Using all these inequalities for $f_{1}$, we get, $\max \left(f_{1}(x)\right)_{\alpha}=x^{t} U_{\alpha}(\tilde{C}) x+x U_{\alpha}(\tilde{E})+U_{\alpha}(\tilde{\gamma})$.

Similarly, $\min \left(f_{2}(x)\right)_{\alpha}=x^{t} L_{\alpha}(\tilde{D}) x+x L_{\alpha}(\tilde{F})+L_{\alpha}(\tilde{\beta}) \Rightarrow \max (\tilde{f}(x))_{\alpha}=\frac{x^{t} U_{\alpha}(\tilde{C}) x+x U_{\alpha}(\tilde{E})+U_{\alpha}(\tilde{\gamma})}{x^{t} L_{\alpha}(\tilde{D}) x+x L_{\alpha}(\tilde{F})+L_{\alpha}(\tilde{\beta})}$.

Particular case: As we know that $(\tilde{z})_{\alpha}=\left[L_{\alpha}(\tilde{z}), U_{\alpha}(\tilde{z})\right]$. For $\lambda \in R$, 
$\lambda\left[L_{\alpha}(\tilde{z}), U_{\alpha}(\tilde{z})\right]=\left\{\begin{array}{l}{\left[\lambda L_{\alpha}(\tilde{z}), \lambda U_{\alpha}(\tilde{z})\right], \lambda \geq 0} \\ {\left[\lambda U_{\alpha}(\tilde{z}), \lambda L_{\alpha}(\tilde{z})\right], \text { otherwise }}\end{array}\right.$

So, if $\lambda=-1$ i.e., coefficient of any term in the function is negative then $U_{\alpha}(\tilde{z})$ must be replaced by $L_{\alpha}(\tilde{z})$ and vice-versa.

\section{Problem Formulation}

In BL-MOFQFOM, both leader and follower try to optimise their objective vector by cooperating with each other and paying major attention to the preferences of other.

Consider the bi-level decision maker's hierarchical system composed of functions $\tilde{F}_{1}$ and $\tilde{F}_{2}$ over $x=\left(x_{1}, x_{2}\right)$ where $x_{1}=\left(x_{1}^{1}, x_{1}^{2}, \ldots, x_{1}^{n_{1}}\right)$ and $x_{2}=\left(x_{2}^{1}, x_{2}^{2}, \ldots, x_{2}^{n_{2}}\right) . \tilde{F}_{1}$ is the leader having control over $x_{1} \in R^{n_{1}}$ and $\tilde{F}_{2}$ is the follower having control over $x_{2} \in R^{n_{2}} . x_{1}, x_{2}$ are the decision variables. Thus, BL-MO fully quadratic fractional optimization model is of the form:

Level1:

$\max _{x_{1}} \tilde{F}_{1}\left(x_{1}, x_{2}\right)=\max _{x_{1}}\left(\tilde{f}_{1}^{1}(x), \tilde{f}_{1}^{2}(x), \ldots, \tilde{f}_{1}^{t_{1}}(x)\right)$

where $x_{1}$ is the vector of decision variable and $x_{1} \in \mathrm{R}^{n_{1}}$,

\section{Level 2:}

$\max _{x_{2}} \tilde{F}_{2}\left(x_{1}, x_{2}\right)=\max _{x_{2}}\left(\tilde{f}_{2}^{1}(x), \tilde{f}_{2}^{2}(x), \ldots, \tilde{f}_{2}^{t_{2}}(x)\right)$

where $x_{2}$ is the vector of decision variable and $x_{2} \in \mathrm{R}^{n_{2}}$,

subject to

$x \in \tilde{S}=\left\{x \in \mathrm{R}^{n} / \tilde{r}_{1} x_{1}^{2}+\tilde{r}_{2} x_{2}^{2}+\tilde{r}_{3} x_{1} x_{2}+\tilde{r}_{4} x_{1}+\tilde{r}_{5} x_{2}+\tilde{r}_{6}\left(\begin{array}{l}\leq \\ = \\ \geq\end{array}\right) \tilde{b} ; x \in[0, \infty), b \in \mathrm{R}^{m}\right\}$,

$\tilde{f}_{i}^{j}(x)=\frac{\tilde{g}_{i}^{j}(x)}{\tilde{h}_{i}^{j}(x)}=\frac{\tilde{a}_{i}^{j} x_{1}^{2}+\tilde{b}_{i}^{j} x_{1} x_{2}+\tilde{c}_{i}^{j} x_{2}^{2}+\tilde{d}_{i}^{j} x_{1}+\tilde{e}_{i}^{j} x_{2}+\tilde{f}_{i}^{j}}{\tilde{p}_{i}^{j} x_{1}^{2}+\tilde{q}_{i}^{j} x_{1} x_{2}+\tilde{r}_{i}^{j} x_{2}^{2}+\tilde{s}_{i}^{j} x_{1}+\tilde{t}_{i}^{j} x_{2}+\tilde{u}_{i}^{j}}$.

where, $\tilde{F}_{i}: R^{n_{1}} \times R^{n_{2}} \rightarrow R^{t_{i}} t_{i}, n_{1}, n_{2}$ are natural numbers; $\mathrm{i}=1,2$ and $1 \leq j \leq t_{i} ; r_{k}=\left[r_{k 1}, r_{k 2}, \ldots, r_{k m}\right]$; and $\tilde{a}_{i}^{j}, \tilde{b}_{i}^{j}, \tilde{c}_{i}^{j}, \tilde{d}_{i}^{j}, \tilde{e}_{i}^{j}, \tilde{f}_{i}^{j}, \tilde{p}_{i}^{j}, \tilde{q}_{i}^{j}, \tilde{r}_{i}^{j}, \tilde{s}_{i}^{j}, \tilde{t}_{i}^{j}, \tilde{u}_{i}^{j}$ are fuzzy vectors and fuzzy numbers like triangular, trapezoidal, and so on. $\quad \tilde{r}_{k} ; 1 \leq k \leq 6$ and $\tilde{b}$ vector is the fuzzy matrix of order $m \times 1$. These fuzzy parameters are characterised by their membership functions. It is common to take $h_{i}^{j}(x)>0 ; x \in S, i=1,2 ; 1 \leq j \leq t_{i}$ and $\tilde{S}$ is the region common to all convex constraints in fuzzy environment. 


\section{Conversion of Fuzzy Models to Crisp by $\alpha$-Cuts}

In BL-MOFQFOM (equation (1)), coefficients involved in model are the fuzzy numbers. Let $\mu_{a_{i}^{j}}, \mu_{b_{i}^{j}}, \mu_{c_{i}^{j}}, \mu_{d_{i}^{j}}, \mu_{e_{i}^{j}}, \mu_{f_{i}^{j}}, \mu_{p_{i}^{j}}, \mu_{q_{i}^{j}}, \mu_{r_{i}^{j}}, \mu_{s_{i}^{j}}, \mu_{t_{i}^{j}}, \mu_{u_{i}^{j}}$ be the membership functions associated with $\tilde{a}_{i}^{j}, \tilde{b}_{i}^{j}, \tilde{c}_{i}^{j}, \tilde{d}_{i}^{j}, \tilde{e}_{i}^{j}, \tilde{f}_{i}^{j}, \tilde{p}_{i}^{j}, \tilde{q}_{i}^{j}, \tilde{r}_{i}^{j}, \tilde{s}_{i}^{j}, \tilde{t}_{i}^{j}, \tilde{u}_{i}^{j}$ respectively. Let membership functions corresponding to $\tilde{r}_{k}, \tilde{b}$ be denoted by $\mu_{\tilde{b}}$ and $\mu_{\tilde{r}_{k}}$ respectively. For any value of $\alpha$ decided between 0 to 1 , define $\alpha$-cut as suggested by El Sayed et al. (2020):

$$
\left(\tilde{a}_{i}^{j}\right)_{\alpha}=\left\{a_{i}^{j} \in\left[L_{\alpha}\left(a_{i}^{j}\right), U_{\alpha}\left(a_{i}^{j}\right)\right] / \mu_{\tilde{a}_{i}^{j}}(x) \geq \alpha \quad x \in S\left(\tilde{a}_{i}^{j}\right)\right\}
$$

and in the similar way, define $\alpha$-cuts for $\tilde{b}_{i}^{j}, \tilde{c}_{i}^{j}, \tilde{d}_{i}^{j}, \tilde{e}_{i}^{j}, \tilde{f}_{i}^{j}, \tilde{p}_{i}^{j}, \tilde{q}_{i}^{j}, \tilde{r}_{i}^{j}, \tilde{s}_{i}^{j}, \tilde{t}_{i}^{j}, \tilde{u}_{i}^{j}$. For a fuzzy number $T, S(T)$ denotes the supports of $T$. Assume that $\tilde{h}_{i}^{j}(x)>0 \forall x$. For desired value of $\alpha$, the upper and lower bounds of level set are used to obtain crisp objective functions. To maximize objective functions, we will use upper and lower bounds of $\alpha$-cut of $\tilde{g}_{i}^{j}(x)$ and $\tilde{h}_{i}^{j}(x)$ respectively, i.e.

$$
\begin{aligned}
& \left(f_{i}^{j}(x)\right) \alpha=\frac{U_{\alpha}\left(g_{i}^{j}(x)\right)}{L_{\alpha}\left(h_{i}^{j}(x)\right)}= \\
& \frac{U_{\alpha}\left(a_{i}^{j}\right) x_{1}^{2}+U_{\alpha}\left(b_{i}^{j}\right) x_{1} x_{2}+U_{\alpha}\left(c_{i}^{j}\right) x_{2}^{2}+U_{\alpha}\left(d_{i}^{j}\right) x_{1}+U_{\alpha}\left(e_{i}^{j}\right) x_{2}+U_{\alpha}\left(f_{i}^{j}\right)}{L_{\alpha}\left(p_{i}^{j}\right) x_{1}^{2}+L_{\alpha}\left(q_{i}^{j}\right) x_{1} x_{2}+L_{\alpha}\left(r_{i}^{j}\right) x_{2}^{2}+L_{\alpha}\left(s_{i}^{j}\right) x_{1}+L_{\alpha}\left(t_{i}^{j}\right) x_{2}+L_{\alpha}\left(u_{i}^{j}\right)} \\
& \quad i=1,2 ; 1 \leq j \leq t_{i}
\end{aligned}
$$

In minimization of objective functions, we will use lower bound of $\alpha$-cut for $\tilde{g}_{i}^{j}(x)$ and upper bound of $\alpha$-cut for $\tilde{h}_{i}^{j}(x)$.

$$
\begin{gathered}
\left(f_{i}^{j}(x)\right)_{\alpha}=\frac{L_{\alpha}\left(g_{i}^{j}(x)\right)}{U_{\alpha}\left(h_{i}^{j}(x)\right)}= \\
\frac{L_{\alpha}\left(a_{i}^{j}\right) x_{1}^{2}+L_{\alpha}\left(b_{i}^{j}\right) x_{1} x_{2}+L_{\alpha}\left(c_{i}^{j}\right) x_{2}^{2}+L_{\alpha}\left(d_{i}^{j}\right) x_{1}+L_{\alpha}\left(e_{i}^{j}\right) x_{2}+L_{\alpha}\left(f_{i}^{j}\right)}{U_{\alpha}\left(p_{i}^{j}\right) x_{1}^{2}+U_{\alpha}\left(q_{i}^{j}\right) x_{1} x_{2}+U_{\alpha}\left(r_{i}^{j}\right) x_{2}^{2}+U_{\alpha}\left(s_{i}^{j}\right) x_{1}+U_{\alpha}\left(t_{i}^{j}\right) x_{2}+U_{\alpha}\left(u_{i}^{j}\right)} \\
i=1,2 ; 1 \leq j \leq t_{i}
\end{gathered}
$$

The constraints set having a convex feasible choice set is represented by $\tilde{S}$ in fuzzy environment and $\alpha$-cut determines a numerical interval which can also be applied to constraints in equation (1) to convert them into deterministic ones. So,

$$
\begin{aligned}
& \tilde{r}_{1 k} x_{1}^{2}+\tilde{r}_{2 k} x_{2}^{2}+\tilde{r}_{3 k} x_{1} x_{2}+\tilde{r}_{4 k} x_{1}+\tilde{r}_{5 k} x_{2}+\tilde{r}_{6 k} \leq \tilde{b}_{k} ; k=1,2, \ldots, m_{1} \\
& \tilde{r}_{1 k} x_{1}^{2}+\tilde{r}_{2 k} x_{2}^{2}+\tilde{r}_{3 k} x_{1} x_{2}+\tilde{r}_{4 k} x_{1}+\tilde{r}_{5 k} x_{2}+\tilde{r}_{6 k} \leq \tilde{b}_{k} ; k=m_{1}+1, m_{1}+2, \ldots, m
\end{aligned}
$$

are replaced by the following constraints: 


$$
\begin{aligned}
& L_{\alpha}\left(r_{1 k}\right) x_{1}^{2}+L_{\alpha}\left(r_{2 k}\right) x_{2}^{2}+L_{\alpha}\left(r_{3 k}\right) x_{1} x_{2}+L_{\alpha}\left(r_{4 k}\right) x_{1}+L_{\alpha}\left(r_{5 k}\right) x_{2}+L_{\alpha}\left(r_{6 k}\right) \leq U_{\alpha}\left(b_{k}\right) \\
& k=1,2, \ldots, m_{1} \\
& U_{\alpha}\left(r_{1 k}\right) x_{1}^{2}+U_{\alpha}\left(r_{2 k}\right) x_{2}^{2}+U_{\alpha}\left(r_{3 k}\right) x_{1} x_{2}+U_{\alpha}\left(r_{4 k}\right) x_{1}+U_{\alpha}\left(r_{5 k}\right) x_{2}+U_{\alpha}\left(r_{6 k}\right) \leq L_{\alpha}\left(b_{k}\right) \\
& k=m_{1}+1, \ldots, m_{2}
\end{aligned}
$$

where, $L_{\alpha}\left(r_{i k}\right) ; 1 \leq i \leq 6$ and $U_{\alpha}\left(r_{i k}\right) ; 1 \leq i \leq 6$ denotes infimum and supremum of $\alpha$-level set of coefficients for all objective functions.

Constraints having an equal sign as in

$$
\begin{gathered}
\tilde{r}_{1 k} x_{1}^{2}+\tilde{r}_{2 k} x_{2}^{2}+\tilde{r}_{3 k} x_{1} x_{2}+\tilde{r}_{4 k} x_{1}+\tilde{r}_{5 k} x_{2}+\tilde{r}_{6 k}=\tilde{b}_{k} \\
k=m_{2}+1, \ldots, m
\end{gathered}
$$

will be replaced by following two equivalent constraints

$$
\begin{gathered}
L_{\alpha}\left(r_{1 k}\right) x_{1}^{2}+L_{\alpha}\left(r_{2 k}\right) x_{2}^{2}+L_{\alpha}\left(r_{3 k}\right) x_{1} x_{2}+L_{\alpha}\left(r_{4 k}\right) x_{1}+L_{\alpha}\left(r_{5 k}\right) x_{2}+L_{\alpha}\left(r_{6 k}\right) \leq U_{\alpha}\left(b_{k}\right) \\
U_{\alpha}\left(r_{1 k}\right) x_{1}^{2}+U_{\alpha}\left(r_{2 k}\right) x_{2}^{2}+U_{\alpha}\left(r_{3 k}\right) x_{1} x_{2}+U_{\alpha}\left(r_{4 k}\right) x_{1}+U_{\alpha}\left(r_{5 k}\right) x_{2}+U_{\alpha}\left(r_{6 k}\right) \leq L_{\alpha}\left(b_{k}\right) \\
k=m_{2}+1, \ldots, m
\end{gathered}
$$

Thus, after setting value of $\alpha$, BL-MOFQFOM is composed of:

Level1:

$$
\max _{x_{1}}\left(F_{1}(x)\right)_{\alpha}=\max _{x_{1}}\left(f_{1}^{1}(x)_{\alpha}, f_{1}^{2}(x)_{\alpha}, \ldots, f_{1}^{t_{1}}(x)_{\alpha}\right)
$$

$x=\left(x_{1}, x_{2}\right)$ and $x_{1}$ is the vector of decision variable $x_{1} \in R^{n_{1}}$,

Level 2:

$$
\left.\max _{x_{2}}\left(F_{2}(x)\right)_{\alpha}=\max _{x_{2}}\left(f_{2}^{1}(x)_{\alpha}, f_{2}^{2}(x)_{\alpha}, \ldots, f_{2}^{t_{2}}(x)_{\alpha}\right)\right)
$$$$
x=\left(x_{1}, x_{2}\right) \text { and } x_{2} \text { is the vector of decision variable } x_{2} \in R^{n_{2}} \text {, }
$$

$$
\begin{array}{r}
U_{\alpha}\left(r_{1 k}\right) x_{1}^{2}+U_{\alpha}\left(r_{2 k}\right) x_{2}^{2}+U_{\alpha}\left(r_{3 k}\right) x_{1} x_{2}+U_{\alpha}\left(r_{4 k}\right) x_{1}+U_{\alpha}\left(r_{5 k}\right) x_{2}+U_{\alpha}\left(r_{6 k}\right) \geq L_{\alpha}\left(b_{k}\right) \\
k=1,2, \ldots, m_{1}, m_{2}+1, \ldots, m \\
L_{\alpha}\left(r_{1 k}\right) x_{1}^{2}+L_{\alpha}\left(r_{2 k}\right) x_{2}^{2}+L_{\alpha}\left(r_{3 k}\right) x_{1} x_{2}+L_{\alpha}\left(r_{4 k}\right) x_{1}+L_{\alpha}\left(r_{5 k}\right) x_{2}+L_{\alpha}\left(r_{6 k}\right) \leq U_{\alpha}\left(b_{k}\right) \\
k=m_{1}+1, \ldots, m_{2}, m_{2}+1, \ldots, m
\end{array}
$$

$x \geq 0$.

The common region for constraints in equations (7), (8), (10) and (11) at $\alpha$-level is denoted by $S_{\alpha}$, a compact subset of Real number line.

\section{Proposed Methodology to Solve BL-MOFQOM with FGP Approach for the Decided Value of $\alpha$}


In FGP, our main focus is to obtain satisfactory solution which is acceptable to all decisionmakers by using Pareto-optimality.

\subsection{Membership Functions for Objective Functions and Decision Variables}

The vectors of objective functions of both leader and follower are identified by their membership functions at the given $\alpha$-level. $\alpha$-cut method has been extensively used by methodologists to reduce fuzzy model into crisp one. Let $\mu_{\left(f_{1}^{j}\right)_{\alpha}} ;\left(1 \leq j \leq t_{1}\right)$ be the vector of membership function of the leader and $\left(\mu_{\left(f_{2}^{j}\right)_{\alpha}}\right) ;\left(1 \leq j \leq t_{2}\right)$ be the vector of membership function of follower. To define these membership functions at $\alpha$-level, each objective function's maximum and minimum are taken individually that represent aspiration and tolerance levels. $\operatorname{Max}_{x}\left(f_{i}^{j}(x)\right)_{\alpha} i=1,2 ; 1 \leq j \leq t_{i}$ stands for the maximum value of objective function. $\operatorname{Min}_{x}\left(f_{i}^{j}(x)\right)_{\alpha} i=1,2 ; 1 \leq j \leq t_{i}$ denotes the minimum value of the objective function $\left(f_{i}^{j}(x)\right)_{\alpha}$.
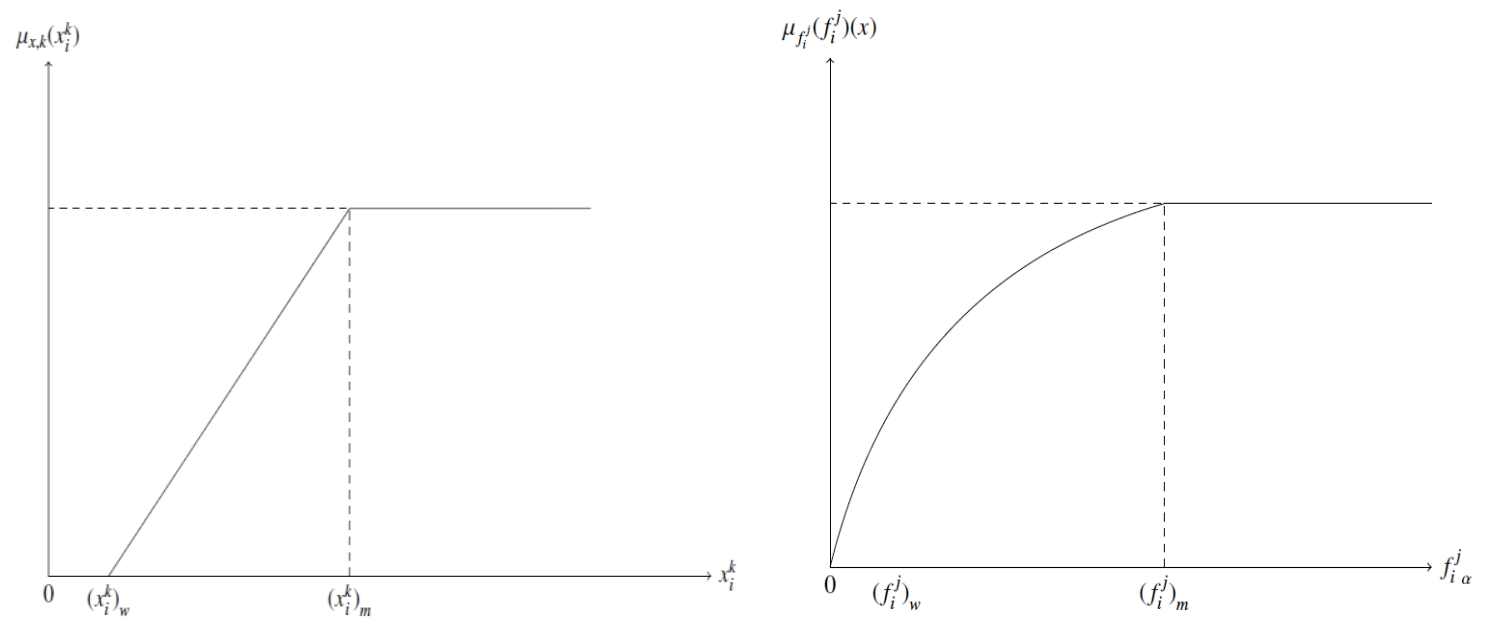

Figure 1. Membership functions for $x_{i}^{k}$ and $f_{i}^{j}$.

To define membership function, each objective function's lower tolerance limit and upper tolerance limit are decided by using best and worst values at the given $\alpha$-level. Let $\left(f_{i}^{j}\right)_{M}$ be the upper limit of tolerance or wished level of the membership function of objective function $\left(f_{i}^{j}\right)$. Similarly, let $\left(f_{i}^{j}\right)_{W}$ be the lower limit of tolerance of achievement for the membership function of $f_{i}^{j}(x) ; i=1,2 ; 1 \leq j \leq t_{i}$ objective function. Any value of $\left(f_{i}^{j}(x)\right)_{\alpha} \geq\left(f_{i}^{j}\right)_{M}\left(i=1,2 ; 1 \leq j \leq t_{i}\right)$ is highly aspired but any value of the function $\left(f_{i}^{j}(x)\right)_{\alpha} \leq\left(f_{i}^{j}\right)_{W}\left(i=1,2 ; 1 \leq j \leq t_{i}\right)$ is completely unacceptable. So the membership function, as shown graphically in Figure 1, which characterises fuzzy goal is defined as: 
$\mu_{f_{i}^{j}}\left(f_{i}^{j}(x)\right)_{\alpha}= \begin{cases}0 & \left(f_{i}^{j}(x)\right)_{\alpha} \leq\left(f_{i}^{j}\right)_{W} \\ \frac{\left(f_{i}^{j}(x)\right)_{\alpha}-\left(f_{i}^{j}\right)_{W}}{\left(f_{i}^{j}\right)_{M}-\left(f_{i}^{j}\right)_{W}} & \left(f_{i}^{j}\right)_{W} \leq\left(f_{i}^{j}\left(x_{1}\right)_{\alpha} \leq\left(f_{i}^{j}\right)_{M} i=1,2 ; 1 \leq j \leq t_{i}\right. \\ 1 & \left(f_{i}^{j}(x)\right)_{\alpha} \geq\left(f_{i}^{j}\right)_{M}\end{cases}$

Decision variable's membership function of the leader is considered as

$$
\mu_{x_{1}^{k}}\left(x_{1}^{k}\right)= \begin{cases}0 & x_{1}^{k} \leq\left(x_{1}^{k}\right)_{W} \\ \frac{x_{1}^{k}-\left(x_{1}^{k}\right)_{W}}{\left(x_{1}^{k}\right)_{M}-\left(x_{1}^{k}\right)_{W}} & \left(x_{1}^{k}\right)_{W} \leq x_{1}^{k} \leq\left(x_{1}^{k}\right)_{M} K=1,2, \ldots, n_{1} \\ 1 & x_{1}^{k} \geq\left(x_{1}^{k}\right)_{M}\end{cases}
$$

where, $\left(x_{1}^{k}\right)_{w}$ and $\left(x_{1}^{k}\right)_{M}$ are the vectors in $R^{n_{1}}$ which yield minimum and maximum values of the objective functions of leader.

\subsection{Fuzzy Goal Programming Approach}

In FGP, every DM aims to maximise their objective function. The maximum value of every objective function calculated individually becomes the aspired level. For membership function defined in equations (13) and (14), the membership goal for aspiration level as unity is expressed as

$\mu_{f_{i}^{j}}\left(f_{i}^{j}(x)\right)_{\alpha}+u_{i j}-v_{i j}=1 ; i=1,2 ; 1 \leq j \leq t_{i}$

and

$\mu_{x_{i}^{k}}\left(x_{i}^{k}\right)_{\alpha}+\dot{u}_{i k}-\dot{v}_{i k}=1 ; i=1,1 \leq k \leq n$

or equivalent, as

$\frac{\left(f_{i}^{j}(x)\right)_{\alpha}-\left(f_{i}^{j}\right)_{W}}{\left(f_{i}^{j}\right)_{M}-\left(f_{i}^{j}\right)_{w}}+u_{i j}-v_{i j}=1 ; i=1,2 ; 1 \leq j \leq t_{i}$

and

$\frac{\left(x_{1}^{k}\right)_{\alpha}-\left(x_{1}^{k}\right)_{w}}{\left(x_{1}^{k}\right)_{m}-\left(x_{1}^{k}\right)_{w}}+\dot{u}_{1 k}-\dot{v}_{1 k}=1 ; 1 \leq k \leq n_{1}, k \in N$

where, $u_{i j}, \dot{u}_{1 k}$ represents the under deviations and $v_{i j} \dot{v}_{1 k}$ represents the over deviations from the aspired levels, $i=1,2 ; 1 \leq j \leq t ; 1 \leq k \leq n_{1}$ and $u_{i j}, \dot{u}_{1 k}, v_{i j}, \dot{v}_{1 k} \geq 0$. Now objective becomes to minimise the values of under deviational variables for the higher value of membership function. To maximise the achievement of leader and follower, we will make a linear combination of under deviational variables with scalars as their weights. Over deviational variable shows the complete achievement of the fuzzy goal. Thus, the final $\alpha$-(BL-MOFQFOM) of the problem is 
$\operatorname{Min} Z=\sum_{i=1}^{2} \sum_{j=1}^{t_{i}} W_{i j}^{-} u_{i j}+\sum_{k=1}^{n_{1}} W_{1 k}\left(\dot{u}_{1 k}+\dot{v}_{1 k}\right)$

subject to

$$
\begin{aligned}
& \frac{\left(f_{i}^{j}(x)\right)_{\alpha}-\left(f_{i}^{j}\right)_{W}}{\left(f_{i}^{j}\right)_{M}-\left(f_{i}^{j}\right)_{W}}+u_{i j}-v_{i j}=1 ; i=1,2 ; 1 \leq j \leq t_{i} \\
& \frac{x_{1}^{k}-\left(x_{1}^{k}\right)_{W}}{\left(x_{1}^{k}\right)_{M}-\left(x_{1}^{k}\right)_{W}}+\dot{u}_{1 k}-\dot{v}_{1 k}=1 ; k=1,2, \ldots, n_{1} \\
& U_{\alpha}\left(r_{1 k}\right) x_{1}^{2}+U_{\alpha}\left(r_{2 k}\right) x_{2}^{2}+U_{\alpha}\left(r_{3 k}\right) x_{1} x_{2}+U_{\alpha}\left(r_{4 k}\right) x_{1}+U_{\alpha}\left(r_{5 k}\right)+U_{\alpha}\left(r_{6 k}\right) \geq L_{\alpha}\left(b_{k}\right) \text {; } \\
& k=1,2, \ldots, m_{1}, m_{2}+1, \ldots, m . \\
& L_{\alpha}\left(r_{1 k}\right) x_{1}^{2}+L_{\alpha}\left(r_{2 k}\right) x_{2}^{2}+L_{\alpha}\left(r_{3 k}\right) x_{1} x_{2}+L_{\alpha}\left(r_{4 k}\right) x_{1}+L_{\alpha}\left(r_{5 k}\right)+L_{\alpha}\left(r_{6 k}\right) \leq U_{\alpha}\left(b_{k}\right) \text {; } \\
& k=m_{1}+1, \ldots, m_{2}, m_{2}+1, \ldots, m \text {. }
\end{aligned}
$$

$u_{i j} \times v_{i j}=0$ and $u_{i j}, v_{i j} \geq 0 ; i=1,2 ; 1 \leq j \leq t_{i}$,

$\dot{u}_{1 k} \times \dot{v}_{1 k}=0$ and $\dot{u}_{1 k}, \dot{v}_{1 k} \geq 0 ; k=1,2, \ldots, n_{1}$,

where, $W_{i j}^{-}=\frac{1}{\left(f_{i}^{j}\right)_{M}-\left(f_{i}^{j}\right)_{W}} ; i=1,2 ; 1 \leq j \leq t_{i}$

$W_{1 k}=\frac{1}{\left(x_{1}^{k}\right)_{M}-\left(x_{1}^{k}\right)_{W}} ; k=1,2, \ldots, n_{1}$.

are the numerical weights which play a vital role in achieving the aspired level of fuzzy goals.

\subsection{Simplification of Fuzzy Goals}

As we are dealing with quadratic fractional objective function, so the membership goals which we obtain in Equation (17) will be complex and may arise computational difficulties. To avoid such a problem, we will apply a process to convert membership function into a simpler form. So, we consider fuzzy goals as:

$$
\begin{aligned}
& \mu_{f_{i}^{j}}\left(f_{i}^{j}(x)\right)_{\alpha}+u_{i j}-v_{i j}=1 \\
& \text { i.e. } \frac{\left(f_{i}^{j}(x)\right)_{\alpha}-\left(f_{i}^{j}(x)\right)_{w}}{\left(f_{i}^{j}(x)\right)_{M}-\left(f_{i}^{j}(x)\right)_{w}}+u_{i j}-v_{i j}=1
\end{aligned}
$$

Put the value of $\left[\left(f_{i}^{j}(x)\right)_{M}-\left(f_{i}^{j}(x)\right)_{W}\right]^{-1}$ as defined in equation (19). We get,

$$
\begin{aligned}
& W_{i j}^{-}\left[\left(f_{i}^{j}(x)\right)_{\alpha}-\left(f_{i}^{j}(x)\right)_{w}\right]+u_{i j}-v_{i j}=1 \\
& W_{i j}^{-}\left(f_{i}^{j}(x)\right)_{\alpha}-W_{i j}^{-}\left(f_{i}^{j}(x)\right)_{W}+u_{i j}-v_{i j}=1
\end{aligned}
$$


$W_{i j}^{-} \frac{\left(U_{\alpha}\left(g_{i}^{j}(x)\right)\right)}{\left(L_{\alpha}\left(h_{i}^{j}(x)\right)\right)}-W_{j i}^{-}\left(f_{i}^{j}(x)\right)_{W}+u_{i j}-v_{i j}=1$

$W_{i j}^{-} U_{\alpha}\left(g_{i}^{j}(x)\right)-W_{i j}^{-}\left(f_{i}^{j}(x)\right)_{W} L_{\alpha}\left(h_{i}^{j}(x)\right)+L_{\alpha}\left(h_{i}^{j}(x)\right) u_{i j}-L_{\alpha}\left(h_{i}^{j}(x)\right) v_{i j}=L_{\alpha}\left(h_{i}^{j}(x)\right)$

Put

$L_{\alpha}\left(h_{i}^{j}(x)\right) u_{i j}=\dot{U}_{i j}^{-}$

$L_{\alpha}\left(h_{i}^{j}(x)\right) v_{i j}=\dot{V}_{i j}$

$\Rightarrow W_{i j}^{-} U_{\alpha}\left(g_{i}^{j}(x)\right)-W_{i j}^{-}\left(f_{i}^{j}(x)\right)_{w} L_{\alpha}\left(h_{i}^{j}\right)+\dot{U}_{i j}-\dot{V}_{i j}=L_{\alpha}\left(h_{i}^{j}\right)$

is the simpler form of fuzzy goal equation (21) where $\dot{U}_{i j}, \dot{V}_{i j} \geq 0$ because $u_{i j}, v_{i j} \geq 0$ and $L_{\alpha}\left(h_{i}^{j}\right)$ is greater than $0 ; i=1,2 ; 1 \leq j \leq t_{i}$. Previously our objective was to minimize $u_{i j}$ moduled into non-linear objective including $\dot{U}_{i j}$ as defined in equation (26). It is clear that $u_{i j}=0$ stands for full achievement of fuzzy goal and $u_{i j}=1$ stands for zero achievement of fuzzy goal, so $u_{i j} \leq 1$ implies

$\frac{\dot{U}_{i j}}{L_{\alpha}\left(h_{i}^{j}\right)(x)} \leq 1$

$\dot{U}_{i j} \leq L_{\alpha}\left(h_{i}^{j}(x)\right), i=1,2 ; 1 \leq j \leq t_{i}$

which become constraints in the final model. Thus our new model consists of $\dot{U}_{i j}$ in place of $u_{i j}$ is detailed as:

$\min Z=\sum_{i=1}^{2} \sum_{j=1}^{t_{i}} W_{i j}^{-} \dot{U}_{i j}+\sum_{k=1}^{n_{1}} \mathrm{~W}_{1 k}\left(\dot{u}_{1 k}+\dot{v}_{1 k}\right)$

$U_{\alpha}\left(r_{1 k}\right) x_{1}^{2}+U_{\alpha}\left(r_{2 k}\right) x_{2}^{2}+U_{\alpha}\left(r_{3 k}\right) x_{1} x_{2}+U_{\alpha}\left(r_{4 k}\right) x_{1}+U_{\alpha}\left(r_{5 k}\right) x_{2}+U_{\alpha}\left(r_{6 k}\right) \geq L_{\alpha}\left(b_{k}\right)$

$k=1,2, \ldots, m_{1}, m_{2}+1, \ldots, m$

$L_{\alpha}\left(r_{1 k}\right) x_{1}^{2}+L_{\alpha}\left(r_{2 k}\right) x_{2}^{2}+L_{\alpha}\left(r_{3 k}\right) x_{1} x_{2}+L_{\alpha}\left(r_{4 k}\right) x_{1}+L_{\alpha}\left(r_{5 k}\right)+L_{\alpha}\left(r_{6 k}\right) \leq U_{\alpha}\left(b_{k}\right)$;

$k=m_{1}+1, \ldots, m_{2}, m_{2}+1, \ldots, m$

$\dot{U}_{i j} \leq L_{\alpha}\left(h_{i}^{j}\right)$ 


$$
\begin{aligned}
& \frac{x_{1}^{k}-\left(x_{1}^{k}\right)_{W}}{\left(x_{1}^{k}\right)_{M}-\left(x_{1}^{k}\right)_{w}}+\dot{u}_{1 k}-\dot{v}_{1 k}=1 \\
& W_{i j}^{-} U_{\alpha}\left(g_{i}^{j}\right)-W_{i j}^{-}\left(f_{i}^{j}\right)_{w} L_{\alpha}\left(g_{i}^{j}\right)+\dot{U}_{i j}-\dot{V}_{i j}=L_{\alpha}\left(h_{i}^{j}\right) \\
& i=1,2 ; 1 \leq j \leq t_{i} ; 1 \leq k \leq n_{1} \\
& x \geq 0, \dot{U}_{i j} \geq 0, \dot{V}_{i j} \geq 0, \dot{u}_{1 k}, \dot{v}_{1 k} \geq 0 \\
& \dot{u}_{i j} \times \dot{v}_{i j}=0 \text { and } \dot{U}_{i j} \times \dot{V}_{i j}=0
\end{aligned}
$$

This model provides us a compromised solution of BL-MOFQOM in the fuzzy environment for the desired value of $\alpha$.

\section{Algorithm BL-MOFQOM with Fuzzy Demands}

This section presents the algorithm to specify the proposed approach.

\section{Algorithm}

Step 1: In the shadow of decision makers, assign a acceptable value to $\alpha$.

Step 2: Use the equations (2)-(12) to change the model into deterministic form.

Step 3: Calculate the greatest and smallest values of each objective functions of leader and follower both.

Step 4: Establish aspiration $\left(f_{i}^{j}\right)_{M}$ and tolerance limit $\left(f_{i}^{j}\right)_{W}$ at an upper and lower level.

Step 5: Use equation (14) to define membership functions for decision variable of leader.

Step 6: Use equation (13) to define membership function for decision vector.

Step 7: Calculate all the weights $W_{i j}^{-}$and $W_{1 k}$.

Step 8: Use equations (15)-(18) to formulate fuzzy goals.

Step 9: Apply equations (20)-(30) to simplify fuzzy goals and construct the FGP final model (equation (31)) for $\alpha$-(BL-MOFQFOM).

Step 10: Solve obtained model and if the compromised solution is accepted by all decisionmakers, then go to step 12 if not, then go to step 11.

Step 11: Alter values of $\left(f_{i}^{j}\right)_{M}$ and $\left(f_{i}^{j}\right)_{W}$ then move to step 4 .

Step 12: Terminate with the acceptable compromised solution.

Flowchart: This section presents the flowchart (Figure 2) to clarify the proposed method. 


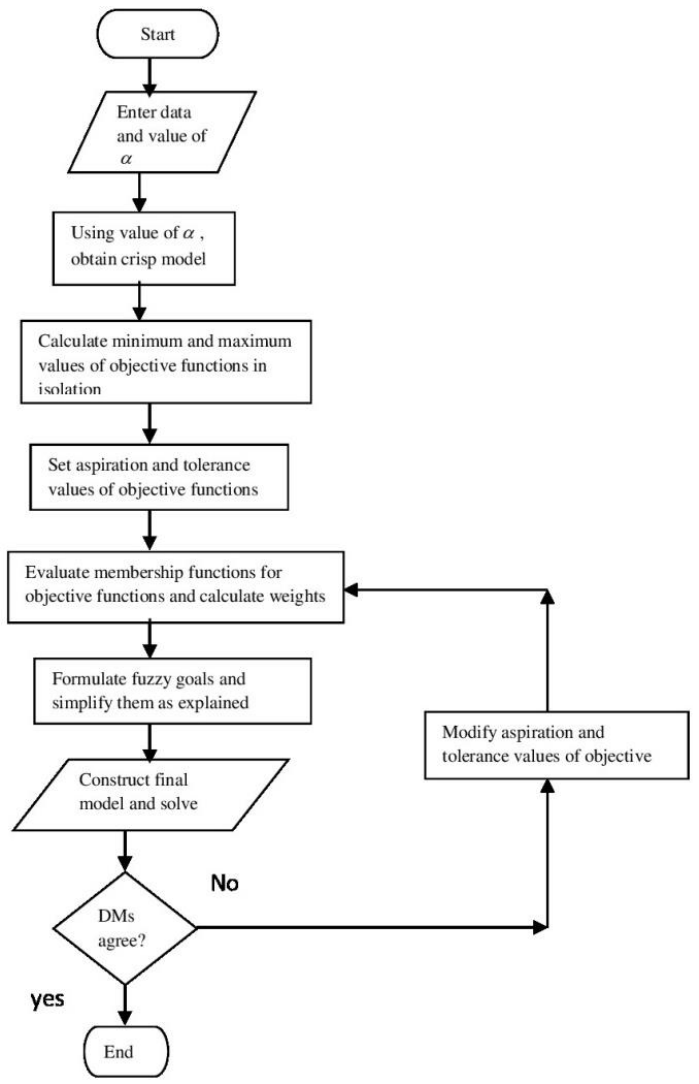

Figure 2. Flowchart to explain procedure.

\section{Numerical Illustration}

\section{Numerical 1:}

Consider here BL-MOFQOM to explain the practicability of the propounded method.

Level1:

$\max _{x_{1}}\left(\frac{0 . \tilde{8} x_{1}^{2}-\tilde{2} x_{2}^{2}+\tilde{5} x_{2}+1 \tilde{0}}{\tilde{1} x_{1}^{2}+\tilde{3} x_{2}+\tilde{5}}, \frac{-\tilde{2} x_{1}^{2}-\tilde{1} x_{2}^{2}+\tilde{5} x_{1}+\tilde{8}}{\tilde{1} x_{1}^{2}+\tilde{3} x_{2}+\tilde{5}}\right)$

where $x_{2}$ solves,

Level 2:

$\max _{x_{2}}\left(\frac{-\tilde{1} x_{1}^{2}-\tilde{2} x_{2}^{2}+\tilde{6}}{\tilde{1} x_{2}^{2}+\tilde{3} x_{1}+\tilde{1}}, \frac{-\tilde{3} x_{1}^{2}-\tilde{1} x_{2}^{2}+\tilde{8}}{\tilde{1} x_{2}^{2}+\tilde{3} x_{1}+\tilde{1}}\right)$,

subject to

$\tilde{1} x_{1}^{2}+\tilde{2} x_{1}+\tilde{7} x_{2} \leq 1 \tilde{5}$,

$\tilde{1} x_{2}^{2}+\tilde{3} x_{1}+2 . \tilde{2} x_{2} \leq 1 \tilde{8}$

$\tilde{1} x_{2}^{2}+0 . \tilde{8} x_{1}+\tilde{7} x_{2} \geq \tilde{8}$,

$x_{1} \geq 0, x_{2} \geq 0$. 
and triangular fuzzy numbers are given as $0 . \tilde{8}=(0.8,0.8,0.8), \tilde{1}=(0,1,2), \tilde{2}=(2,3,4)$, $\tilde{3}=(2,3,4) \quad, \quad \tilde{5}=(4,5,6) \quad, \quad \tilde{6}=(5.5,6,6.5) \quad, \quad \tilde{7}=(6,7,8) \quad, \quad \tilde{8}=(7,8,9) \quad, \quad 1 \tilde{0}=(9,10,10)$, $1 \tilde{5}=(14,15,16), 1 \tilde{8}=(17,18,19)$.

\section{Solution:}

Step 1: First, assume 0.8 value of $\alpha$ is accepted by all DMS.

Step 2: Deterministic model of the given problem corresponding to $0.8=\alpha$ is obtained as

Level1:

$\max _{x_{1}}\left(\frac{0.8 x_{1}^{2}-1.8 x_{2}^{2}+5.2 x_{2}+9.8}{0.8 x_{1}^{2}+2.8 x_{2}+4.8}, \frac{-1.8 x_{1}^{2}-0.8 x_{2}^{2}+5.2 x_{1}+8.1}{0.8 x_{1}^{2}+2.8 x_{2}+4.8}\right)$

where $x_{2}$ solves.

Level 2:

$\max _{x_{2}}\left(\frac{-0.8 x_{1}^{2}-1.8 x_{2}^{2}+6.1}{0.8 x_{2}^{2}+2.8 x_{1}+0.8}, \frac{-2.8 x_{1}^{2}-0.8 x_{2}^{2}+8.1}{0.8 x_{2}^{2}+2.8 x_{1}+0.8}\right)$,

subject to

$0.8 x_{1}^{2}+1.8 x_{1}+6.8 x_{2} \leq 15.2$

$0.8 x_{2}^{2}+2.8 x_{1}+2.2 x_{2} \leq 18.2$,

$1.2 x_{2}^{2}+0.8 x_{1}+7.2 x_{2} \geq 7.9$,

$x_{1} \geq 0, x_{2} \geq 0$.

Step (3)-(4): Individual maximum and minimum of all DMs and lower and upper tolerance level of objective function are described in Table 1 .

Table 1. Individual highest and lowest values of DMS with aspiration and tolerance limits.

\begin{tabular}{|c|c|c|c|c|}
\hline Values & $\left(f_{1}^{1}(x)\right)_{0.8}$ & $\left(f_{2}^{1}(x)\right)_{0.8}$ & $\left(f_{1}^{2}(x)\right)_{0.8}$ & $\left(f_{2}^{2}(x)\right)_{0.8}$ \\
\hline max. & 1.76 & 1.36 & 2.953 & 4.86 \\
\hline min. & 1.123 & 0.370 & -0.603 & -1.3720 \\
\hline$\left(f_{i}^{j}\right)_{M}$ & 1.77 & 1.37 & 2.954 & 4.9 \\
\hline$\left(f_{i}^{j}\right)_{W}$ & 1.1 & 0.3 & -0.604 & -1.4 \\
\hline
\end{tabular}

Step (5)-(6): Select all $\mu_{f_{j}^{i}}\left(f_{i}^{j}\right)_{\alpha}$ and $\mu_{x_{1}^{k}}\left(x_{1}^{k}\right)_{\alpha}$ where $i=1,2 ; 1 \leq j \leq t_{i} ; 1 \leq k \leq n_{1}$. Step (7): Finding all the weights $W_{i j}^{-}$and $W_{1 k}$ as defined in Table 2.

Table 2. Weights of DMs functions and variables.

\begin{tabular}{|c|c|c|c|c|}
\hline$W_{11}^{-}$ & $W_{12}^{-}$ & $W_{21}^{-}$ & $W_{22}^{-}$ & $W_{11}$ \\
\hline 1.49 & 0.934 & 0.28 & 0.158 & 4.1 \\
\hline
\end{tabular}


Step (8)-(9): So the final model for BL-MOFQOM with fuzzy demands is obtained as:

$$
\begin{aligned}
& \text { min } Z=1.49 \dot{U}_{11}+0.934 \dot{U}_{12}+0.28 \dot{U}_{21}+0.158 \dot{U}_{22}+4.1\left(\dot{u}_{1 k}+\dot{v}_{1 k}\right) \\
& \text { subject to } \\
& -0.616 x_{2}^{1}-1.8 x_{2}^{2}+0.244 x_{2}+\dot{U}_{11}-\dot{V}_{11}=1.304, \\
& -2.896 x_{1}^{2}-0.8 x_{2}^{2}+5.2 x_{1}-3.836 x_{2}+\dot{U}_{12}-\dot{V}_{12}=-1.1524, \\
& -0.8 x_{1}^{2}-4.1632 x_{2}^{2}-8.2712 x_{1}+\dot{U}_{21}-\dot{V}_{21}=-3.7368, \\
& -2.8 x_{1}^{2}-6.96 x_{2}^{2}-13.72_{1}+\dot{U}_{22}-\dot{V}_{22}=4.18 \\
& -0.536 x_{1}^{2}-1.876 x_{2}+\dot{U}_{11} \leq 3.216 \\
& -0.856 x_{1}^{2}-2.996 x_{2}+\dot{U}_{12} \leq 5.136 \\
& -2.8464 x_{2}^{2}-9.9624 x_{1}-\dot{U}_{21} \leq 2.8464, \\
& -5.04 x_{2}^{2}-17.64 x_{1}-\dot{U}_{22} \leq 5.04 \\
& 4.166 x_{1}+\dot{u}_{11}-\dot{v}_{11}=5.04 \\
& 0.8 x_{1}^{2}+1.8 x_{1}+6.8 x_{2} \leq 15.2, \\
& 0.8 x_{2}^{2}+2.8 x_{1}+2.2 x_{2} \leq 18.2, \\
& 1.2 x_{2}^{2}+0.8 x_{1}+7.2 x_{2} \geq 7.9, \\
& x_{1} \geq 0, x_{2} \geq 0 \\
& \dot{U}_{11}, \dot{V}_{11}, \dot{U}_{12}, \dot{V}_{12}, \dot{U}_{21}, \dot{V}_{21}, \dot{U}_{22}, \dot{V}_{22}, \dot{u}_{11}, \dot{v}_{11} \geq 0 \\
& \dot{u}_{11} \times \dot{v}_{11}=0, \dot{U}_{i j} \times \dot{V}_{i j}=0, i, j=1,2 .
\end{aligned}
$$

Lingo software version 15 has been used to obtain the compromised solution of 0.8-(BLMOFQOM) with $\left(x_{1}, x_{2}\right)=(0.243,0.926)$ and values of objectives as $\left(f_{1}^{1}\right)_{0.8}=1.76,\left(f_{2}^{1}\right)_{0.8}=1.152,\left(f_{2}^{1}\right)_{0.8}=1.152$ and $\left(f_{2}^{2}\right)_{0.8}=3.35$. Membership functions corresponding to these objectives are $\mu_{f_{1}^{1}}=0.985, \mu_{f_{2}^{1}}=0.79, \mu_{f_{1}^{2}}=0.76, \mu_{f_{2}^{2}}=0.75$.

\section{Numerical 2:}

Level1:

$$
\max _{x_{1}}\left\{\begin{array}{c}
\frac{(0.8,0.9,0.9) x_{1}^{2}+(1,1.2,1.4) x_{2}^{2}}{(0.3,0.4,0.5) x_{1}^{2}+(1.85,1.9,2) x_{2}+(2.1,2.2,2.3)}, \\
\frac{(1,1,1) x_{1}+(1.5,1.7,1.8) x_{2}^{2}+(0.9,1,1)}{(0.9,1,1.1) x_{1}^{2}+(0.3,0.4,0.45) x_{2}^{2}+(3.5,3.6,3.7)}
\end{array}\right\},
$$

Level 2:

$$
\max _{x_{2}}\left\{\begin{array}{l}
\frac{(0.9,1,1) x_{1}^{2}+(0.7,0.8,0.9) x_{2}+(4.7,4.8,4.9)}{(1,1.1,1.2) x_{2}^{2}+(0.8,0.9,1) x_{2}+(2.9,3,3.1)} \\
\frac{(1,1.1,1.2) x_{1}^{2}+(1,1,1) x_{2}^{2}+(1.8,1.9,2)}{(1,1.1,1.2) x_{1}+(1,1.2,1.4) x_{2}^{2}}
\end{array}\right\},
$$


subject to

$(2.1,2.2,2.2) x_{1}^{2}+(2.5,2.6,2.7) x_{2} \leq(5,5,5)$,

$(0.7,0.8,0.9) x_{1} x_{2}+(1.5,1.6,1.7) x_{1} \geq(1.7,1.8,1.9)$,

$(2,2,2) x_{1}^{2}+(0.8,0.9,0.9) x_{2}^{2} \leq(3,3.1,3.1)$,

$x_{1} \geq 0, x_{2} \geq 0$.

Solution: On solving the above problem as explained in the methodology for $\alpha=0.6$, the crisp model is extracted as:

Level1:

$\max _{x_{1}}\left\{\frac{0.9 x_{1}^{2}+1.32 x_{2}^{2}}{0.36 x_{1}^{2}+1.88 x_{2}+2.16}, \frac{x_{1}+1.74 x_{2}^{2}+1}{0.96 x_{1}^{2}+0.36 x_{2}^{2}+3.56}\right\}$,

Level 2:

$\max _{x_{2}}\left\{\frac{x_{1}^{2}+0.84 x_{2}+4.84}{1.06 x_{2}^{2}+0.86 x_{2}+2.96}, \frac{1.14 x_{1}^{2}+x_{2}^{2}+1.94}{1.06 x_{1}+1.16 x_{2}^{2}}\right\}$

subject to

$2.16 x_{1}^{2}+2.56 x_{2} \leq 5$

$0.84 x_{1} x_{2}+1.64 x_{1} \geq 1.76$,

$2 x_{1}^{2}+0.84 x_{2}^{2} \leq 3.1$,

$x_{1} \geq 0, x_{2} \geq 0$.

Next step is to find the individual maximum and minimum of all objective functions as shown in Table 3.

Table 3. Individual highest and lowest values of all objective functions with optimal points.

\begin{tabular}{|c|c|c|c|c|}
\hline Values & $\left(f_{1}^{1}(x)\right)_{0.6}$ & $\left(f_{2}^{1}(x)\right)_{0.6}$ & $\left(f_{1}^{2}(x)\right)_{0.6}$ & $\left(f_{2}^{2}(x)\right)_{0.6}$ \\
\hline max. & 0.735 at $(0.578,1.67)$ & 1.317 at $(0.578,1.67)$ & 2.158 at $(1.244,0)$ & 18.194 at $(1.068,0.008))$ \\
\hline min. & 0.289 at $(0.887,0.407)$ & 0.444 at $(1.064,0.0157)$ & 0.894 at $(0.578,1.67)$ & 1.535 at $(0.578,1.67)$ \\
\hline
\end{tabular}

Using all these values as aspiration and tolerance values, membership functions are formed and afterwards, fuzzy goals are constructed which appear as the constraints in the final model as:

$$
\begin{aligned}
& \min z=2.242 d_{1}^{-}+1.145 d_{2}^{-}+0.7911 d_{3}^{-}+0.06 d_{4}^{-}+3.236\left(p_{1}+s_{1}\right) \\
& \text { subject to } \\
& 0.6354 x_{1}^{2}+1.32 x_{2}^{2}-1.3813 x_{2}+d_{1}^{-}-d_{1}^{+}=1.587, \\
& -1.264 x_{1}^{2}+1.2652 x_{2}^{2}+x_{1}+d_{2}^{-}-d_{2}^{+}=3.687, \\
& x_{1}^{2}-2.2868 x_{2}^{2}-1.015 x_{2}+d_{3}^{-}-d_{3}^{+}=1.5472, \\
& 1.14 x_{1}^{2}-20.103 x_{2}^{2}-16.035 x_{1}+d_{4}^{-}-d_{4}^{+}=-1.94,
\end{aligned}
$$




$$
\begin{aligned}
& d_{1}^{-} \leq 0.16 x_{1}^{2}+0.838 x_{2}+0.96336 \\
& d_{2}^{-} \leq 0.838 x_{1}^{2}+0.314 x_{2}^{2}+3.107, \\
& d_{3}^{-} \leq 1.339 x_{2}^{2}+1.087 x_{2}+3.74, \\
& d_{4}^{-} \leq 17.6596 x_{1}+19.3256 x_{2}^{2}, \\
& x_{1}+p_{1}-s_{1}=0.887 \\
& 2.16 x_{1}^{2}+2.56 x_{2} \leq 5 \\
& 0.84 x_{1} x_{2}+1.64 x_{1} \geq 1.76 \\
& 2 x_{1}^{2}+0.84 x_{2}^{2} \leq 3.1 \\
& x_{1} \geq 0, x_{2} \geq 0
\end{aligned}
$$

After solving the above model, we get optimal point $(1.07,0)$. This optimal point is the efficient solution of the initial model, and on putting the obtained points together and the values of objective functions become $f_{1}^{1}=0.4, f_{1}^{2}=0.444, f_{2}^{1}=2.02$ and $f_{2}^{2}=2.87$ which are all near to their aspired values.

\section{Conclusions}

On analysing above explained examples, it is clear that the utmost efforts are being made to get the calculated values of all objective functions near aspiration values for the betterment of association which justifies the proposed approach. Throughout this paper, we have solved BLMOFQOM subject to Quadratic constraints by using fuzzy goal programming. In the presented approach, the membership functions are made simple to avoid errors due to calculations. The highly compromised solution is provided by deciding the $\alpha$-level which can be changed according to the requirements of DM's. An attempt is being made to reveal the concept to solve BL-MOFQOM with fuzzy demands which are appealed by the organisations such as engineering, banking, transportation, management, economic system and supply chain planning. This approach can be extended further to solve the tri-level multi-objective and multi-level multi-objective optimization problems.

\section{Conflict of Interest}

The authors confirm that there is no conflict of interest to declare for this publication.

\section{Acknowledgments}

This research did not receive any specific grant from funding agencies in the public, commercial, or not-for-profit sectors. The authors would like to thank the editor and anonymous reviewers for their comments which helps to improve the quality of this work.

\section{References}

Abo-Sinna, M.A., \& Baky, I.A. (2007). Interactive balance space approach for solving multi-level multiobjective programming problems. Information Sciences, 177(16), 3397-3410.

Ammar, E.E. (2008). On solutions of fuzzy random multiobjective quadratic programming with applications in portfolio problem. Information Sciences, 178(2), 468-484. 
Baky, I.A. (2009). Fuzzy goal programming algorithm for solving decentralized bi-level multi-objective programming problems. Fuzzy Sets and Systems, 160(18), 2701-2713.

Baky, I.A. (2010). Solving multi-level multi-objective linear programming problems through fuzzy goal programming approach. Applied Mathematical Modelling, 34(9), 2377-2387.

Baky, I.A., Eid, M.H., \& El Sayed, M.A. (2014). Bi-level multi-objective programming problem with fuzzy demands: a fuzzy goal programming algorithm. Opsearch, 51(2), 280-296.

Candler, W., \& Townsley, R. (1982). A linear two-level programming problem. Computers \& Operations Research, 9(1), 59-76.

El Sayed, M.A., Baky, I.A., \& Singh, P. (2020). A modified TOPSIS approach for solving stochastic fuzzy multi-level multi-objective fractional decision making problem. Opsearch, 57(4), 1374-1403.

Elsisy, M.A., \& El Sayed, M.A. (2019). Fuzzy rough bi-level multi-objective nonlinear programming problems. Alexandria Engineering Journal, 58(4), 1471-1482.

Júdice, J.J., \& Faustino, A.M. (1992). A sequential LCP method for bilevel linear programming. Annals of Operations Research, 34(1), 89-106.

Kumar, S., \& Rakshit, M. (2017, October). Interactive fuzzy programming procedure for trilevel quadratic fractional programming. In 2017 International Conference on Computing and Communication Technologies for Smart Nation (IC3TSN) (pp. 243-248). IEEE. Gurgaon, India.

Mishra, S., \& Ghosh, A. (2006). Interactive fuzzy programming approach to bi-level quadratic fractional programming problems. Annals of Operations Research, 143(1), 251-263.

Osman, M.S., Emam, O.E., \& El Sayed, M.A. (2018a). Solving multi-level multi-objective fractional programming problems with fuzzy demands via FGP approach. International Journal of Applied and Computational Mathematics, 4(1), 41.

Osman, M.S., Emam, O.E., \& Elsayed, M.A. (2018b). Interactive approach for multi-level multi-objective fractional programming problems with fuzzy parameters. Beni-Suef University Journal of Basic and Applied Sciences, 7(1), 139-149.

Osman, M.S., Emam, O.E., Raslan, K.R., \& Farahat, F.A. (2018c). Solving bi-level multi-objective quadratic fractional programming problems in rough environment through FGP approach. Journal of Abstract and Computational Mathematics, 3(1), 6-21.

Pal, B.B., \& Moitra, B.N. (2003). A fuzzy goal programming procedure for solving quadratic bilevel programming problems. International Journal of Intelligent Systems, 18(5), 529-540.

Pradhan, A., \& Biswal, M.P. (2019). Linear fractional programming problems with some multi-choice parameters. International Journal of Operational Research, 34(3), 321-338.

Shih, H.S., Lai, Y.J., \& Lee, E.S. (1996). Fuzzy approach for multi-level programming problems. Computers \& Operations Research, 23(1), 73-91.

Zadeh, L.A. (1965). Information and control. Fuzzy Sets, 8(3), 338-353.

Original content of this work is copyright @International Journal of Mathematical, Engineering and Management Sciences. Uses under the Creative Commons Attribution 4.0 International (CC BY 4.0) license at https://creativecommons.org/licenses/by/4.0/ 